\title{
Bococizumab for the treatment of hypercholesterolaemia
}

\author{
Nicola Ferri ${ }^{a}$, Alberto Corsini ${ }^{b, c}$, Cesare R. Sirtorid and Massimiliano Ruscica ${ }^{b}$ \\ aDepartment of Pharmaceutical and Pharmacological Sciences, Università di Padova, Padoua, Italy; bipartimento di Scienze Farmacologiche e \\ Biomolecolari, Università degli Studi di Milano, Milan, Italy; 'Multimedica IRCCS, Milano, Italy; ${ }^{d}$ Centro Dislipidemie, A.S.S.T. Grande Ospedale \\ Metropolitano Niguarda, Milan, Italy
}

ABSTRACT

Introduction: Low-density lipoprotein cholesterol (LDL-C) remains a well-established risk factor for cardiovascular disease (CVD). LDL-C levels are considered primary targets of therapy. A new series of systemic biomolecules, the monoclonal antibodies (mAbs) of proprotein convertase subtilisin/kexin type 9 (PCSK9), have a higher activity in reducing LDL-C.

Areas covered: The authors critically review the current evidence on the efficacy and safety of bococizumab, a humanized mAb against PCSK9, which was surprisingly discontinued in November 2016. The pharmacokinetic profile and the biological features of bococizumab vs others mAbs are also discussed. As of now, in adjunct to diet, alirocumab and evolocumab are the only approved PCSK9 $\mathrm{mAbs}$ for the treatment of adult patients with severe clinical atherosclerotic CVD already at maximallytolerated statin therapy and require additional LDL-C lowering.

Expert opinion: Although discontinued, data from a phase $2 \mathrm{~b}$ trial show the effectiveness of bococizumab in lowering LDL-C in a similar way to the two available PCSK9 antagonists. However, some peculiar biological characteristics of bococizumab may explain the attenuation of LDL-C lowering over time, as well as a higher rate of immunogenicity and of injection-site reactions.
ARTICLE HISTORY

Received 22 November 2016

Accepted 4 January 2017

\section{KEYWORDS}

Bocucizumab; familial hypercholesterolaemias; LDL-C; humanized monoclonal antibody; PCSK9

\section{Introduction}

Hyperlipidemias, multifactorial conditions partly genetically and partly life habit induced, represent the most important underlying risk factors for cardiovascular disease [1]. The primary effort in hypolipidemic drug therapy is focused on lowering the primary carriers of cholesterol, namely low-density lipoproteins (LDLs) [2]. Among drugs primarily reducing LDL-cholesterol (LDL-C), the monoclonal antibodies (mAbs) against proprotein convertase subtilisin/kexin protein 9 (PCSK9), alirocumab and evolocumab, have been approved in the United States and in the European Union in August 2015 [3]. As of now, the best characterized activity of secreted PCSK9 is to posttranslationally regulate the number of cell-surface LDL receptors (LDL-R) [4]. The PCSK9 gene, coded on chromosome $1 \mathrm{p} 32.3$, is ubiquitously expressed. It binds to the LDL-R, thus not allowing the uptake of LDL particles from extracellular milieu into cells [5]. By this mechanism, PCSK9 raises LDL-C levels.

In adjunct to diet, PCSK9 inhibitors are indicated for the treatment of adult patients with severe clinical atherosclerotic cardiovascular disease (i.e. heart attacks or strokes), who are already at maximally tolerated statin therapy and requiring additional LDL-C lowering [6,7]. The efficacy of PCSK9 inhibitors is dramatic and may reach up to $55 \%$ LDL-C lowering as monotherapy [8-10] and up to $61 \%$ when added to standard therapy $[11,12]$. Of note, the currently approved indications report that heterozygous familial hypercholesterolemic (HeFH) and homozygous familial hypercholesterolemic (HoFH) patients may be treated with evolocumab [13], whereas alirocumab may be prescribed only to HeFH patients [14].

\subsection{Overview of current developments}

Alongside with evolocumab (Repatha - Amgen) and alirocumab (Praluent - Sanofi and Regeneron Pharmaceuticals), bococizumab (also called RN316/PF-04950615; Pfizer) had undergone a large Phase 3 program called 'Studies of PCSK9 Inhibition and the Reduction of vascular Events (SPIRE)' [15]. In a statement issued on 1 November 2016, Pfizer surprisingly announced discontinuation of global development of bococizumab, its investigational PCSK9 mAb.

Furthermore, based on the data from these three compounds, data on the safety and efficacy of the novel agent RN317 (PF-05335810; Pfizer) have been reported from a Phase 1 randomized study. This humanized $\mathrm{mAb}$ is an engineered $\operatorname{lgG} 2 \Delta$ that binds to PCSK9 with a lower affinity at low $\mathrm{pH}$ (acidic endosomal compartment) compared to neutral $\mathrm{pH}$ (plasma). The $\mathrm{pH}$-sensitive binding to PCSK9 prolongs the entity of RN317 half-life extending the duration of LDL-C reduction over an 85-day dosing interval. RN317 appears to escape endosomal degradation and, thus, can be recycled and potentially reutilized [16].

Safety and efficacy data of LY3015014 (Lilly) have been reported in a Phase 2 study [17]. LGT209 (Novartis) has been discovered as a lipid lowering agent but licensed to another
AQ3

AQ4

AQ5 55 


\section{Box 1. Drug summary}

Drug name (generic) Phase (for indication under discussion) Indication (specific to discussion)

Pharmacology description/ mechanism of action

Route of administration Pivotal trial(s) - please name and describe
Bococizumab (RN316/PF-04950615)

Discontinued 1 November 2016

Hypercholesterolemia

Humanized $\lg G 2 \Delta$ a monoclonal antibody that recognizes and binds to the lowdensity lipoprotein receptor (LDL-R)binding domain of PCSK9, thus preventing PCSK9-mediated degradation of LDL-R

Subcutaneous injection Q14

NCT01592240; LDL-cholesterol was significantly reduced by $53.4 \mathrm{mg} / \mathrm{dL}$ at the dose of $150 \mathrm{mg}$ every 2 weeks and by $44.9 \mathrm{mg} / \mathrm{dL}$ at the dose of $300 \mathrm{mg}$ every 4 weeks company (CYON) for the treatment of sepsis, whereas RG7652 (Roche/Genentech) was discontinued in 2014 [18].

Another approach to a possible antagonism to PCSK9 may be by RNA interference (ALN-PCSSC - Alynam) [19] whose single-dose administration led to a rapid and dose-dependent reduction in plasma PCSK9 protein. Higher doses of ALN-PCS resulted in more prolonged PCSK9 reduction [19]. The treatment was shown to be safe with similar adverse events between ALN-PCSsc and placebo groups [19].

A similar interference may be obtained with a PCSK9 binding small molecule (Adnectin; Bristol-Myers Squibb R\&D Company), for which only animal data are available [20].

Finally, vaccination against PCSK9 represents a potential approach to reduce the administration to once a year injections; participants are being recruited to Phase 1 clinical trials of two vaccine candidates (ATH04A and ATH06A by AFFiRiS).

\section{Chemistry}

Bococizumab $\left(\mathrm{C}_{6414} \mathrm{H}_{9918} \mathrm{~N}_{1722} \mathrm{O}_{2012} \mathrm{~S}_{54}\right)$ is a $145.1 \mathrm{~kg} / \mathrm{mol}$ humanized $\mathrm{mAb} \mathrm{G} 2 \Delta \mathrm{a}$, with gamma2 heavy chain (1-444) [humanized VH (Homo sapiens IGHV1-46*01 (90.80\%)-(IGHD)IGHJ1*01 L123>T (113)) [8.8.11] (1-118) -H. sapiens IGHG2*01

95 (CH1 (119-216), hinge (217-228), CH2 A115>S (327), P116>S (328, 229-337), CH3 (338-442), CHS (443-444)) (119-444)], (132-214')-disulfide with kappa light chain $\left(1^{\prime}-214^{\prime}\right)$ [humanized V-KAPPA (H. sapiens IGKV1-13*02 (91.00\%) - IGKJ2*01) [6.3.9] $\left(1^{\prime}-107^{\prime}\right)-H$. sapiens IGKC*01 $\left.\left(108^{\prime}-214^{\prime}\right)\right]$; dimer $(220$ $\left.220^{\prime}: 221-221^{\prime}: 224-224^{\prime}: 227-227^{\prime}\right)$-tetrakisdisulfide.

Bococizumab is a humanized mAb against PCSK9; this feature makes it different from the other two mAbs anti-PCSK9, alirocumab and evolocumab, both are fully human antibodies. Although the incidence of immune reactions to mAbs is usually limited (from $<1 \%$ to $10 \%$ ) [21], the immunoreactivity potential decreases from the first generation of chimeric antibodies to the last series of fully human antibodies $[22,23]$. Indeed, if compared with chimeric and humanized mAbs, they generally show reduced immunogenicity [24]. Anti-bococizumab antibodies were detected in approximately $7 \%$ of treated patients after 24 weeks of administration. One patient ( $0.4 \%$ of total) also developed a partial reduction of the hypocholesterolemic effect of bococizumab, suggesting a potential antagonized effect of endogenous antibodies developed during therapy by the immune system [25].

The development of autoantibodies anti-mAbs has also been registered with both evolocumab and alirocumab, although with a lower incidence. In the DESCARTES study, 2 out of 901 patients had detectable binding antibodies before or at the time of randomization and 1 had transient antievolocumab-binding antibodies after 52 weeks of treatment [8]. No neutralizing antibodies were observed in any treated patient [8]. In the LAPLACE-2 trial, 1899 patients were treated with evolocumab. Three evolocumab-treated patients were positive for binding antibodies before starting treatment. Of these, one had detectable binding antibodies at the end of the study. No cases of neutralizing antibodies were reported [26]. By combining all clinical trials, only $0.1 \%$ of the patients ( 7 out of 4846 with primary or mixed hypercholesterolemia; and 0 out of 80 with familial homozygous hypercholesterolemia) were found positive for anti-evolocumab antibodies.

In a pool of 10 placebo- and active-controlled trials, $4.8 \%$ of patients treated with alirocumab showed antidrug antibodies newly detected after initiating treatment, as compared to $0.6 \%$ of controls. In addition, $1.2 \%$ of patients developed neutralizing antibodies on at least one occasion and $0.3 \%$ exhibited transient or prolonged loss of efficacy [14].

As such, bococizumab appears to be more immunogenic in comparison to alirocumab and evolocumab with higher incidence of developing antidrug antibodies with neutralizing activity. However, it must be taken into account that immunogenicity data are highly dependent on the sensitivity and specificity of the assay. Generally, two-site (bridging) assays and radioimmunoassay are the most common techniques to measure immunogenicity [27]. Thus, the lack of standardized methodologies to measure immunogenicity makes any comparative considerations meaningless [27].

It should be noted that, in the case of the most frequently used monoclonals, i.e. for the treatment of rheumatoid arthritis, a very high prevalence of antimonoclonal antibodies has been reported [28]. Regarding adalimumab, up to $35 \%$ of patients develop circulating antibodies, and the effect of such response on the therapeutic efficacy is still controversial, with studies that show no effect [28], and others reported that antidrug antibodies are significant predictors of no response [29]. Thus, it is conceivable to conclude that the inability to maintain longterm and durable cholesterol lowering of bococizumab may be not exclusively related to the development of antidrug antibodies, but other mechanisms could be envisioned.

A second consideration on the biochemical properties of bococizumab is related to its IgG isotype. Both bococizumab and evolocumab are $\lg G 2-\lambda$, whereas alirocumab is an $\lg G 1$. Although the pharmacological implications of these differences still need to be determined, Yooet al. showed that antibodies of the human IgG2 subclass can form covalent dimers in vivo and that $\lg G 2$ dimers occur in human serum 
$[30,31]$. In the oncological field, the $\lg G 1$ has been the most popular choice for the development of mAbs, mainly due to the fact that antibodies designed for selective eradication of cancer cells typically require an active isotype that permits complement activation and effector-mediated cell killing by antibody-dependent cell-mediated cytotoxicity.

The IgG1 identity can meet all of the criteria of cellmediated cytotoxicity, i.e. by interacting with the complement factor $\mathrm{C} 1$ and with the FcyRI [32], whereas IgG2 does not possess this property. If the therapeutic goal is neutralization of a soluble antigen, such as PCSK9, effector functions are less relevant, and both $\lg G 1$ and $\lg G 2$ can be utilized. Nevertheless, the presence of effector functions in alirocumab (IgG1), compared to evolocumab and bococizumab (both lgG2), still needs to be investigated.

Conversely, the Fcy receptors are expressed on a wide variety of cells, including monocytes, macrophages, dendritic cells, neutrophils, natural killer cells, B cells, and hepatocytes. Thus, based on their innate roles in immune responses, Fcy receptors may be involved in the clearance of alirocumabPCSK9 complexes from cells opsonized by the mAb [33]. However, the significance of Fcy receptor-mediated catabolism of mAbs is unclear [33].

\section{3. Pharmacodynamics}

Bococizumab was selected across $800 \mathrm{mAbs}$ from hybridoma cell lines, generated by fusing the spleen of wild-type and PCSK9 null mice immunized with human PCSK9 protein [34]. The antibodies that positively bound PCSK9 by an ELISA assay were then tested for their effect on the LDL-R degradation in the liver cell line Huh7 incubated with recombinant PCSK9 [34]. Screening led to the identification of four antibodies produced by the hybridoma, completely blocking the activity of PCSK9 on the LDL-R and one of them (J10) showed approximate affinities of $0.3,0.5$, and $2.7 \mathrm{nM}$ with recombinant human, cynomolgus monkey, and mouse PCSK9. J10 also completely and dose-dependently inhibited the binding of human PCSK9 to the LDL-R with an $\mathrm{IC}_{50}$ of $1 \mathrm{nM}$ [34]. $\mathrm{J} 10$ was then engineered into a human $\lg G 2 \triangle \mathrm{A}$ and $\mathrm{K}$ chain antibody for further improving its antigen-binding affinity. From these changes, the J16 antibody containing the fully human sequence outside of the complementarity-determining regions was obtained. J16 binds to a recombinant human PCSK9 molecule with a $\mathrm{Kd}$ of approximately 5 pM, a cynomolgus monkey PCSK9 with a $\mathrm{Kd}$ of less than $100 \mathrm{pM}$, and a mouse PCSK9 with a $\mathrm{Kd}$ of 35 pM. $J 16$ completely blocks the human and mouse PCSK9 binding to the LDL-R [34].

The co-crystal structure of the Fab region of $\mathrm{J} 16$ and recombinant PCSK9 showed that the binding site is located mainly within the catalytic domain of PCSK9, involved in the interaction with the LDL-R. In particular, J16 binds the epitope that almost perfectly overlaps with the LDL-R EGF-A domain binding site on PCSK9 [35]. Both light and heavy chains mediate the interaction between J16 and PCSK9 and the surface covered by the antibody is approximately $788 \AA^{2}$, a larger area than occupied by the LDL-R EGF-A domain $\left(456 \AA^{2}\right)[34,36]$.

\section{Pharmacokinetics and metabolism}

The pharmacokinetic (PK) profile of bococizumab has been recently reported [16]. Bococizumab, when administered to hypercholesterolemic subjects on statin therapy as subcutaneous (s.c.) or intravenous (i.v.) injections, shows a slow absorption. Following single s.c. administrations, $T_{\max }$ values equal to 156 and $71 \mathrm{~h}$ for doses of 1 and $3 \mathrm{mg} / \mathrm{kg}$ are recorded. The maximum concentrations $\left(C_{\max }\right)$ were 11.85 and $1.824 \mu \mathrm{g} / \mathrm{mL}$ for 1 and $3 \mathrm{mg} / \mathrm{kg}$, respectively. Following $C_{\max }$ a multiphasic decline over time was observed, with mean terminal $t_{1 / 2}$ values of $8.4 \pm 2.7$ days at the $3 \mathrm{mg} / \mathrm{kg}$ dose. Thus, the half-life of bococizumab appears to be significantly shorter than those of evolocumab and alirocumab (1117 and 17-20 days, respectively) $[13,14]$.

As expected for a $\mathrm{mAb}$, the steady-state volume of distribution $\left(V_{s s}\right)$ of bococizumab is 17.6 I, i.e. a relatively small value, due to the limited capacity of mAbs to diffuse into different tissues [22]. Absolute bioavailability for bococizumab $1.0 \mathrm{mg} /$ $\mathrm{kg}$ s.c., compared with bococizumab i.v. injection, is $12.5 \%$ [16]. Bioavailability is $44.6 \%$ for bococizumab $(3.0 \mathrm{mg} / \mathrm{kg}$ ) s.c. using dose-normalized AUC for i.v. (bococizumab $1.0 \mathrm{mg} / \mathrm{kg}$ ) as the reference [16].

A PK/pharmacodynamic (PD) model has been developed in order to characterize the relationship among bococizumab dose, plasma concentrations, and LDL-C response [37]. The data for this analysis were derived from a Phase $2 b$ clinical trial [25]. A two-compartment PK model with parallel firstorder and Michaelis-Menten kinetics was linked to an indirect response model describing $L D L-C$ response. Clinical trial simulation indicated a robust reduction in LDL-C and even greater LDL-C lowering if no dose titration was implemented in the Phase $2 b$ study [25]. This analysis clearly indicates that the PK profile of bococizumab follows a two-compartment model with parallel first-order and Michaelis-Menten elimination. A similar PD profile between alirocumab, evolocumab, and bococizumab after the biweekly administration indicates a similar persistence of the antibodies in the circulation.

Although the PK interactions of mAbs and small-molecule drugs are limited, it is tempting to speculate that the clearance of bococizumab, similar to alirocumab and evolocumab, increases in the presence of the coadministration of statins $[13,14]$. Indeed, statins can induce PCSK9 expression facilitating the cellular uptake and clearance of mAbs anti-PCSK9 by the cells [22]. This interaction was not considered clinically relevant for both alirocumab and evolocumab. No dose adjustment being required in the case of statin coadministration $[13,14]$.

\section{Clinical efficacy}

\subsection{Phase 1 studies}

Seven Phase 1 clinical studies have been registered, none of which as yet published, as a full article, in peer-reviewed journals. In diet-managed hypercholesterolemic subjects (LDL-C $\geq 130 \mathrm{mg} / \mathrm{dL}$ ), ascending single i.v. administration of bococizumab (dose range $0.3-18 \mathrm{mg} / \mathrm{kg}$ ) resulted in mean percentage LDL-C reductions from baseline of up to $84 \%$ 
(18 mg/kg). As far as adverse events are concerned, s.c. injections of $2 \mathrm{~mL}$ bococizumab (100 or $200 \mathrm{mg}$ ) gave the highest mean pain score of $3.82 \pm 7.75 \mathrm{~mm}$ (visual analog scale ranging from no pain [0] to $100 \mathrm{~mm}$ ) [38].

NCT00991159 evaluated safety and tolerability of single, escalating, i.v. infusions to healthy adults. NCT01243151 evaluated the safety and tolerability of repeated doses in volunteers with hypercholesterolemia. NCT01435382 estimated the absolute bioavailability of bococizumab in subjects with hypercholesterolemias, not on lipid-lowering therapy. NCT02043301 studied the single-dose PK of bococizumab after s.c. injection into the abdomen, upper arm, or thigh. NCT02458209 assessed the s.c. PK and PD of bococizumab in healthy adults by comparing drug substances manufactured at two different locations and administration via prefilled syringe vs. prefilled pen. NCT01163851 evaluated the PK and PD of a single dose of bococizumab in volunteers on stable atorvastatin doses; the trial website reports the obtained data. NCT01163838 was stopped prior to enrollment.

\subsection{Phase 2 studies}

Before publication of a full manuscript [25], data on Phase 2a studies were presented in abstract forms; i.e. in 135 subjects with a mean baseline LDL-C of $123 \mathrm{mg} / \mathrm{dL}$, already at highdose statins (atorvatstain or simvastain 40 or $80 \mathrm{mg}$ or rosuvastatin 20 or $40 \mathrm{mg}$ ), a 12-week treatment with 3 and $6 \mathrm{mg} /$ $\mathrm{kg}$ of bococizumab, administered i.v. every 4 weeks, gave LDL$\mathrm{C}$ reductions of $46 \%$ and $56 \%$ [38,39].

The NCT01592240, a Phase 2 multicenter, double-blind, placebo-controlled, dose-ranging trial in 351 adults (299 completed treatment) with baseline fasting $L D L \geq 80 \mathrm{mg} / \mathrm{dL}$ and triglycerides $\leq 400 \mathrm{mg} / \mathrm{dL}$, reported no $\mathrm{CV}$ events within 6 months. Randomized subjects received every 14 days s.c. placebo or bococizumab 50,100, or $150 \mathrm{mg}$, alternatively every 28 days s.c. placebo or bococizumab 200 or $300 \mathrm{mg}$. The trial foresaw a peculiar dose-ranging design in which an

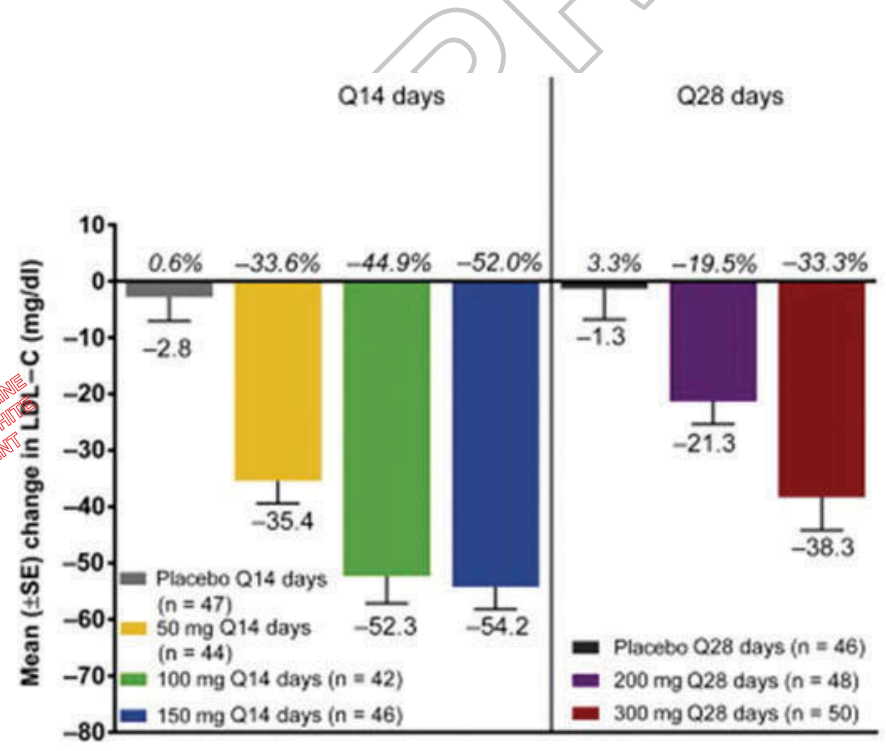

Figure 1. Effect of bococizumab on mean absolute change from baseline in LDL-C following Q14 and Q28 days administrations. Reproduced by permission of Elsevier [25].
LDL-C fall to $\leq 25 \mathrm{mg} / \mathrm{dL}$ corresponded to a reduction in bococizumab dose administration. The primary end point was the absolute change in LDL-C from baseline to week 12 after randomization; 14 days bococizumab $150 \mathrm{mg}$ (Q14) and bococizumab $300 \mathrm{mg}$ (Q28) were the most effective doses. After placebo-adjustment, LDL-C and TC were significantly reduced by $53.4 \mathrm{mg} / \mathrm{dL}$ (Figure 1) and by $53.1 \mathrm{mg} / \mathrm{dL}$ (Q14) and by $44.9 \mathrm{mg} / \mathrm{dL}$ and by $45.6 \mathrm{mg} / \mathrm{dL}$ (Q28), respectively. Non-HDL-C and apolipoprotein (apoB), further markers of CV risk, were significantly lowered by 55.6 and $32.1 \mathrm{mg} / \mathrm{dL}$ (Q14) and by 48.6 and $28.5 \mathrm{mg} / \mathrm{dL}$ (Q28), respectively. ApoB can be considered as equivalent to non-HDL-C especially in case of hypertriglyceridemias [2]. HDL-C levels were significantly raised by $6.5 \%(p=0.043)$ in the Q28 arm. Regardless of doses, median percentage changes in TG showed a trend wise to reduction. Median percentage reductions of $L p(a)$ were $9 \%$ and $10.7 \%$ at Q14 and Q28, respectively. ApoA-I increments were not statistically significant, 6.5\% (Q14) and 2.7 (Q28). No differences were recorded for serious adverse events across the placebo and bococizumab arms [25]. Nasopharyngitis and upper respiratory tract infections were found the most frequently adverse events with no differences between the placebo and bococizumab arms; diarrhea, bronchitis, arthralgia, and injection-site reactions were the others. Seven patients (2\%) discontinued treatment [25].

Overall, the Q14 administration was more effective than the monthly one, and it has been applied to the Phase 3 studies.

Notably, after administration of bococizumab, a significant reduction of LDL-C was observed at week 2 with a gradual increase in the 2-week post-nadir. Similar results were observed with evolocumab [40] and alirocumab [41], suggesting similar clearance rates among mAbs.

\subsection{Phase 3 studies}

These studies were halted after the company evaluation of the first results of a 52-week trial on the effects of bococizumab in lowering LDL-C. Pfizer announced the discontinuation of the late-stage PCSK9 inhibitor by a press release on 1 November 2016. Specifically, the company's website reports 'Pfizer Inc. announced today the discontinuation of the global clinical development program for bococizumab; Pfizer has observed an emerging clinical profile that includes an unanticipated attenuation of LDL-C lowering over time, as well as a higher level of immunogenicity and higher rate of injection-site reactions with bococizumab than shown with the other agents in this class' [42].

The SPIRE program was planned to involve more than 30,000 participants worldwide, comprehensive of 6 lipid-lowering studies and 2 cardiovascular outcome trials [43]. All of these trails were aimed at evaluating efficacy, safety, tolerability, magnitude of reduction in atherogenic lipids as well as reduction in the occurrence of major $\mathrm{CV}$ events, namely death due to CVD, myocardial infarction, stroke, and unstable angina requiring urgent revascularization [44].

The following trials were designed to evaluate efficacy of 12-week treatment with bococizumab in lowering LDL-C (measured with a direct assay). SPIRE-HR (NCT01968954) included 727 hyperlipidemic subjects (primary or mixed 
370 dyslipidemia) at risk of CV events, receiving maximally tolerated statin doses; SPIRE-LDL (NCT01968967) enrolled 2139 hyperlipidemic subjects (fasting LDL-C $\geq 70 \mathrm{mg} / \mathrm{dL}$ and triglyceride $\leq 400 \mathrm{mg} / \mathrm{dL}$; high or very high risk of incurring a CV event) on highly effective dose statin; SPIRE-LL (NCT01200514) enrolled 749 hyperlipidemic subjects, treated with a statin, with fasting LDL-C $\geq 100 \mathrm{mg} / \mathrm{dL}$ and triglyceride $\leq 400 \mathrm{mg} / \mathrm{dL}$; SPIRE-AI (NCT02458287) enrolling 299 subjects with hyperlipidemia or dyslipidemia (fasting LDL-C $\geq 70 \mathrm{mg} / \mathrm{dL}$ and triglycerides $\leq 400 \mathrm{mg} / \mathrm{dL}$; treated with a statin) was aimed at evaluating the effect of bococizumab given by an auto-injector; SPIRE-FH (NCT01968980) included $370 \mathrm{HeFH}$ patients.

Since LDL-C reduction, obtained with PCSK9 mAbs, has not been shown yet to reduce clinical CV outcomes, SPIRE-1 (NCT01975376) and SPIRE-2 (NCT01975389) were designed with CV events as their primary outcomes. The enrollment planned to follow about 28,000 high-risk subjects, both in primary and in secondary prevention, across a broad range of CV risk.

\section{Expert opinion}

390 Considering that LDL-C remains the well-established risk factor for CV disease, one of the main outcomes of the SPIRE program was to evaluate if the LDL-C reduction rate driven by bococizumab had some additional benefit among statin-treated and statin-intolerant patients, either in secondary prevention or in high-risk primary prevention [43]. Indeed, the use of PCSK9 mAbs has been, very recently, recommended in (i) very highrisk patients, i.e. those requiring more than a $50 \%$ reduction in LDL-C despite already at maximally tolerated statin plus ezetimibe, (ii) in severe familial hypercholesterolemia (FH) without ASCVD, requiring a further LDL-C reduction, (iii) and in those, already belonging to above groups, who are statin intolerant [6].

The overall evaluation of bococizumab for the management of hypercholesterolemia indicates an effective molecule with a similar activity as the two available PCSK9 antagonists, evolocumab and alirocumab, but with some differences in the type of selected $m A b$, i.e. being a humanized $m A b$. This characteristic may be linked to the occurrence of $\mathrm{mAb}$ antibodies, potentially leading to reduced effectiveness and occurrence of side effects. As pointed out, in the case of $\mathrm{mAbs}$ for rheumatoid arthritis, occurrence of $\mathrm{mAb}$ antibodies is not necessarily linked to reduced effectiveness. This observation, therefore, may lead to a better understanding of the type of antibody selection to be carried out, in order to obtain the best cholesterol response with the least immunogenicity. Pfizer could witness a clinical profile with an unanticipated attenuation of LDL-C lowering over time, as well as a higher level of immunogenicity and a higher rate of injection-site reactions with bococizumab than shown with the other agents in this class [42].

Since occurrence of mAb-targeted antibodies is not necessarily associated with a reduced PD response, the observation of progressive loss of activity of bococizumab may not only be consequent to an increased antibody response. No hard data on specific side effects have been, however, provided. Possibly also for these reasons, trials with bococizumab did not provide any data on the potential for CV outcome prevention, i.e. as expected also for other agents with a similar mechanism. Whether these observations will in some way impact on the safety profile of the two available PCSK9 antibodies, i.e. evolocumab and alirocumab, will require longer and more intensive monitoring. It is within this context that the FOURIER trial was designed to provide important safety data on the long-term administration of evolocumab and achievement of very low LDL-C levels [45]. Particularly, neurocognitive effects of evolocumab are being assessed in the 'Evaluating PCSK9 Binding antiBody Influence oN coGnitive HeAlth in High cardiovascUlar Risk Subjects (EBBINGHAUS)' trial (NCT02207634).

An interesting PD aspect of available PCSK9 antagonists is the lack of a reducing effect on the hs-C-reactive protein, a constant finding after statins $[46,47]$. On the other hand, antibodies can markedly reduce the circulating levels of lipoprotein (a) $[48,49]$, versus essentially no effect of statins. Although the mechanism of the reduction of $L p(a)$ observed during both alirocumab and evolocumab is unclear and debated, a recent finding has reported how PCSK9 does not significantly modulate $L p(a)$ catabolism, but can rather enhance the hepatic generation of $L p(a)$ [50]. Of note, $L p(a)$ reduction driven by bococizumab (-9\% after $150 \mathrm{mg}$ Q14) [25] was lower than that observed with alirocumab (-19.5\% after $150 \mathrm{mg} \mathrm{Q14)} \mathrm{[51]} \mathrm{and}$ evolocumab (-32.3\% after $140 \mathrm{mg} \mathrm{Q12),} \mathrm{respectively} \mathrm{[48].}$

Similar to statins [52], the risk of new-onset diabetes is likely predictable with the use of PCSK9 inhibitors. Although randomized controlled trials with PCSK9 antagonists showed no evidence of increased development of diabetes [53], PCSK9 genetic variants, associated with lower LDL-C, positively correlated to an increased risk of type-2 diabetes mellitus [54]. A $19 \%$ higher risk for diabetes per 1-mmol/L reduction in LDL-C was acknowledged (1.19; 95\% confidence interval, 1.02-1.38) [55]. Notably, the potentially increased risk of new-onset diabetes during treatment with a PCSK9 inhibitor is likely to be confined to subjects with impaired fasting glucose levels [56].

Finally, the high cost of therapy with PCSK9 inhibitors for $\mathrm{FH}$ or atherosclerotic cardiovascular disease patients should be considered. Assuming that the 2015 prices of alirocumab and evolocumab are around $\$ 14,000$ per year, the price should be reduced to $\$ 4536$ or less per patient to be cost-effective on total US healthcare spending [57].

From the failure of the clinical development of bococizumab, one can potentially conclude that long-term treatment with PCSK9 inhibitors should be associated with a very low development of immunogenic mAbs. From available data, only fully human antibodies seem to be suitable for this therapy. Moreover, it should bear in mind that the s.c. injection of mAbs in the presence of antidrug antibodies results in the local formation of immune complexes in the injection site, reducing the release of the drug into the circulation [27].

The development of $\mathrm{pH}$-sensitive mAbs, such as RN317, appears to be a promising venue, also in view of the stability of response, apparently predicting a lower risk of events [16]. In addition, presently available data indicate that the ideal IgG isotype should be the $\lg G 1$, which is less prone to form dimers and to precipitate at the injection site. Unfortunately, the cost of these treatments remains one of the major limiting steps for use 
in the general population. Development of classical small molecules with PCSK9-inhibitory activity appears to be more difficult, but probably an effective and less-expensive approach for generating a new class of hypocholesterolemic drugs.

\section{Funding}

490 M Ruscica is funded by Fondazione Cariplo grant 2015-0552 and intramural grant Linea 2. Azione A received grant by the Università degli Studi di Milano.

\section{Declaration of interest}

A Corsini has received fees for consulting and research funding from Amgen Inc, Sanofi, Pfizer Inc, Mediolanum Farmaceutici, Merck Sharp and Dohme, Mylan and AstraZeneca.

\section{References}

Papers of special note have been highlighted as either of interest $(\cdot)$ or of considerable interest (..) to readers.

1. Yusuf $S$, Hawken $S$, Ounpuu $S$, et al. Effect of potentially modifiable risk factors associated with myocardial infarction in 52 countries (the INTERHEART study): case-control study. Lancet. 2004;364 (9438):937-952.

2. Catapano AL, Graham I, De Backer G, et al. 2016 ESC/EAS guidelines for the management of dyslipidaemias: the task force for the management of dyslipidaemias of the European Society of Cardiology (ESC) and European Atherosclerosis Society (EAS) developed with the special contribution of the European Assocciation for Cardiovascular Prevention \& Rehabilitation (EACPR). Eur Heart J. 2016.

3. McDonagh M, Peterson K, Holzhammer B, et al. A systematic review of PCSK9 inhibitors alirocumab and evolocumab. J Manag Care Spec Pharm. 2016;22(6):641-53q.

4. Ferri N, Corsini A, Macchi C, et al. Proprotein convertase subtilisin kexin type 9 and high-density lipoprotein metabolism: experimental animal models and clinical evidence. Transl Res. 2016;173:19-29.

5. Seidah NG, Prat A. The biology and therapeutic targeting of the proprotein convertases. Nat Rev Drug Discovery. 2012;11(5):367383.

6. Landmesser U, Chapman MJ, Farnier M, et al. European Society of Cardiology/European Atherosclerosis Society Task Force consensus statement on proprotein convertase subtilisin/kexin type 9 inhibitors: practical guidance for use in patients at very high cardiovascular risk. Eur Heart J. 2016.

7. Achimastos A, Alexandrides T, Alexopoulos D, et al. Expert consensus on the rational clinical use of proprotein convertase subtilisin/kexin type 9 (PCSK9) inhibitors. Hormones. 2016;15(1):8-14.

8. Blom DJ, Hala T, Bolognese M, et al. A 52-week placebo-controlled trial of evolocumab in hyperlipidemia. $\mathrm{N}$ Engl $\mathrm{J}$ Med. 2014;370:1809-1819.

9. Koren MJ, Lundqvist $P$, Bolognese $M$, et al. Anti-PCSK9 monotherapy for hypercholesterolemia: the MENDEL-2 randomized, controlled phase III clinical trial of evolocumab. J Am Coll Cardiol. 2014;63(23):2531-2540.

10. Nissen SE, Stroes E, Dent-Acosta RE, et al. Efficacy and tolerability of evolocumab vs ezetimibe in patients with muscle-related statin intolerance: the GAUSS-3 randomized clinical trial. JAMA. 2016;315(15):1580-1590.

11. Robinson JG, Farnier $M$, Krempf $M$, et al. Efficacy and safety of alirocumab in reducing lipids and cardiovascular events. N Engl J Med. 2015;372(16):1489-1499.

12. Sabatine MS, Giugliano RP, Wiviott SD, et al. Efficacy and safety of evolocumab in reducing lipids and cardiovascular events. N Engl J Med. 2015;372(16):1500-1509.
13. EMA. Evolocumab. Summary of product characteristics. [cited 2016 Apr]. Available from: http://wwwemaeuropaeu/docs/en_GB/docu ment_library/EPAR_-_Product_Information/human/003766/ WC500191398pdf

14. EMA. Alirocumab. Summary of product characteristics. [cited 2016 Apr]. http://wwwemaeuropaeu/docs/en_GB/document_library/ EPAR_-_Product_Information/human/003882/WC500194521pdf

15. Valerio MG, Velayati $A$, Jain $D$, et al. Promising new therapies for the treatment of hypercholesterolemia. Expert Opin Biol Ther. 2016;16(5):609-618.

16. Levisetti $M$, Joh $T$, Wan $H$, et al. A phase I randomized study of a specifically engineered, $\mathrm{pH}$-sensitive PCSK9 inhibitor RN317 (PF05335810) in hypercholesterolemic subjects on statin therapy. Clin Transl Sci. 2016.

-. The manuscript shows the data of the pharmacokinetics of bococizumab.

17. Kastelein JJ, Nissen SE, Rader DJ, et al. Safety and efficacy of LY3015014, a monoclonal antibody to proprotein convertase subtilisin/kexin type 9 (PCSK9): a randomized, placebo-controlled phase 2 study. Eur Heart J. 2016;37(17):1360-1369.

18. Joseph L, Robinson JG. Proprotein convertase subtilisin/Kexin type 9 (PCSK9) inhibition and the future of lipid lowering therapy. Prog Cardiovasc Dis. 2015;58(1):19-31.

19. Fitzgerald K, Frank-Kamenetsky M, Shulga-Morskaya S, et al. Effect of an RNA interference drug on the synthesis of proprotein convertase subtilisin/kexin type 9 (PCSK9) and the concentration of serum LDL cholesterol in healthy volunteers: a randomised, single-blind, placebo-controlled, phase 1 trial. Lancet. 2014;383(9911):60-68.

20. Mitchell T, Chao G, Sitkoff D, et al. Pharmacologic profile of the Adnectin BMS-962476, a small protein biologic alternative to PCSK9 antibodies for low-density lipoprotein lowering. J Pharmacol Exp Ther. 2014;350(2):412-424.

21. Hwang WY, Foote J. Immunogenicity of engineered antibodies. Methods. 2005;36(1):3-10.

22. Ferri N, Bellosta $S$, Baldessin $L$, et al. Pharmacokinetics interactions of monoclonal antibodies. Pharmacol Res. 2016;111:592-599.

23. Harding FA, Stickler MM, Razo J, et al. The immunogenicity of humanized and fully human antibodies: residual immunogenicity resides in the CDR regions. mAbs. 2010;2(3):256-265.

24. Nelson AL, Dhimolea E, Reichert JM. Development trends for human monoclonal antibody therapeutics. Nat Rev Drug Discovery. 2010;9(10):767-774.

25. Ballantyne CM, Neutel J, Cropp A, et al. Results of bococizumab, a monoclonal antibody against proprotein convertase subtilisin/ kexin type 9, from a randomized, placebo-controlled, dose-ranging study in statin-treated subjects with hypercholesterolemia. Am J Cardiol. 2015;115(9):1212-1221.

-. This is the only Phase 2 clinical trial showing the efficacy and safety of bococizumab on statin-treated subjects with hypercholesterolemia.

26. Robinson JG, Nedergaard BS, Rogers WJ, et al. Effect of evolocumab or ezetimibe added to moderate- or high-intensity statin therapy on LDL-C lowering in patients with hypercholesterolemia: the LAPLACE-2 randomized clinical trial. JAMA. 2014;311(18):18701882.

27. Keiserman M, Codreanu C, Handa R, et al. The effect of antidrug antibodies on the sustainable efficacy of biologic therapies in rheumatoid arthritis: practical consequences. Expert Rev Clin Immunol. 2014;10(8):1049-1057.

28. Paramarta JE, Baeten DL. Adalimumab serum levels and antidrug antibodies towards adalimumab in peripheral spondyloarthritis: no association with clinical response to treatment or with disease relapse upon treatment discontinuation. Arthritis Res Ther. 2014;16(4):R160.

29. Jani $M$, Chinoy $H$, Warren $R B$, et al. Clinical utility of random antitumor necrosis factor drug-level testing and measurement of antidrug antibodies on the long-term treatment response in rheumatoid arthritis. Arthritis Rheumatol. 2015;67(8):2011-2019.

30. Yoo EM, Wims LA, Chan LA, et al. Human IgG2 can form covalent dimers. J Immunol. 2003;170(6):3134-3138. 
31. Salfeld JG. Isotype selection in antibody engineering. Nat Biotechnol. 2007;25(12):1369-1372.

32. Jefferis R. Antibody therapeutics: isotype and glycoform selection. Expert Opin Biol Ther. 2007;7(9):1401-1413.

33. Dirks NL, Meibohm B. Population pharmacokinetics of therapeutic monoclonal antibodies. Clin Pharmacokinet. 2010;49(10):633-659.

34. Liang H, Chaparro-Riggers J, Strop $P$, et al. Proprotein convertase substilisin/kexin type 9 antagonism reduces low-density lipoprotein cholesterol in statin-treated hypercholesterolemic nonhuman primates. J Pharmacol Exp Ther. 2012;340(2):228-236.

35. Kwon HJ, Lagace TA, McNutt MC, et al. Molecular basis for LDL receptor recognition by PCSK9. Proc Natl Acad Sci USA. 2008;105 (6):1820-1825.

36. Poirier S, Mayer G. The biology of PCSK9 from the endoplasmic reticulum to lysosomes: new and emerging therapeutics to control low-density lipoprotein cholesterol. Drug Des Devel Ther. 2013;7:1135-1148.

37. Wang E, Plowchalk D, Gibiansky L, et al. Population pharmacokinetic and pharmacodynamics modeling of bococizumab (RN316/PF04950615) in hypercholesterolemic subjects \{abstract]. Atherosclerosis. 2014;235:e192-e301.

38. Gumbiner B, Joh T, Udata C, et al. Effects of 12 weeks of treatment with RN316 (PF-04950615), a humanized IgG2 $\Delta$ a monoclonal antibody binding proprotein convertase subtilisin Kexin Type 9, in hypercholesterolemic subjects on high and maximal dose statins. Circulation. 2012;126:2776-2799.

39. Dadu RT, Ballantyne CM. Lipid lowering with PCSK9 inhibitors. Nat Reviews Cardiol. 2014;11(10):563-575.

40. Giugliano RP, Desai NR, Kohli P, et al. Efficacy, safety, and tolerability of a monoclonal antibody to proprotein convertase subtilisin/kexin type 9 in combination with a statin in patients with hypercholesterolaemia (LAPLACE-TIMI 57): a randomised, placebo-controlled, doseranging, phase 2 study. Lancet. 2012;380(9858):2007-2017.

41. McKenney JM, Koren MJ, Kereiakes DJ, et al. Safety and efficacy of a monoclonal antibody to proprotein convertase subtilisin/kexin type 9 serine protease, SAR236553/REGN727, in patients with primary hypercholesterolemia receiving ongoing stable atorvastatin therapy. J Am Coll Cardiol. 2012;59(25):2344-2353.

42. Pfizer discontinues global development of bococizumab, its investigational PCSK9 inhibitor. 2016 [cited 2016 Nov 22]. Available from: http://www.pfizer.com/news/press-release/press-releasedetail/pfizer_discontinues_global_development_of_bococizumab_ its_investigational_pcsk9_inhibitor

43. Ridker PM, Amarenco P, Brunell R, et al. Evaluating bococizumab, a monoclonal antibody to PCSK9, on lipid levels and clinical events in broad patient groups with and without prior cardiovascular events: rationale and design of the studies of PCSK9 inhibition and the reduction of vascular events (SPIRE) lipid lowering and SPIRE cardiovascular outcomes trials. Am Heart J. 2016;178:135-144.

- This manuscrpt describes the rationale and design of SPIRE program for clinical development of bococizumab, including the impact on cardiovascular morbidity and mortality in highrisk patients.

44. Bergeron N, Phan BA, Ding Y, et al. Proprotein convertase subtilisin/ kexin type 9 inhibition: a new therapeutic mechanism for reducing cardiovascular disease risk. Circulation. 2015;132(17):1648-1666.
45. Sabatine MS, Giugliano RP, Keech A, et al. Rationale and design of the further cardiovascular outcomes research with PCSK9 inhibition in subjects with elevated risk trial. Am Heart J. 2016;173:94-101.

46. Sahebkar A, Rathouska J, Derosa G, et al. Statin impact on disease activity and C-reactive protein concentrations in systemic lupus erythematosus patients: a systematic review and meta-analysis of controlled trials. Autoimmun Rev. 2016;15(4):344-353.

47. Ridker PM, Danielson E, Fonseca FA, et al. Reduction in C-reactive protein and LDL cholesterol and cardiovascular event rates after initiation of rosuvastatin: a prospective study of the JUPITER trial. Lancet. 2009;373(9670):1175-1182.

48. Desai NR, Kohli P, Giugliano RP, et al. AMG145, a monoclonal antibody against proprotein convertase subtilisin kexin type 9 , significantly reduces lipoprotein(a) in hypercholesterolemic patients receiving statin therapy: an analysis from the LDL-C assessment with proprotein convertase subtilisin Kexin type 9 monoclonal antibody inhibition combined with statin therapy (LAPLACE)thrombolysis in myocardial infarction (TIMI) 57 trial. Circulation. 2013;128(9):962-969.

49. Gaudet D, Kereiakes DJ, McKenney JM, et al. Effect of alirocumab, a monoclonal proprotein convertase subtilisin/kexin 9 antibody, on lipoprotein(a) concentrations (a pooled analysis of $150 \mathrm{mg}$ every two weeks dosing from phase 2 trials). Am J Cardiol. 2014;114 (5):711-715

50. Villard EF, Thedrez A, Blankenstein J, et al. PCSK9 modulates the secretion but not the cellular uptake of lipoprotein(a) ex vivo. An effect blunted by alirocumab. JACC Basic Transl Sci. 2016;1:419427.

51. Stein EA, Gipe D, Bergeron J, et al. Effect of a monoclonal antibody to PCSK9, REGN727/SAR236553, to reduce low-density lipoprotein cholesterol in patients with heterozygous familial hypercholesterolaemia on stable statin dose with or without ezetimibe therapy: a phase 2 randomised controlled trial. Lancet. 2012;380(9836):29-36.

52. Ruscica M, Macchi C, Morlotti B, et al. Statin therapy and related risk of new-onset type 2 diabetes mellitus. Eur J Intern Med. 2014;25(5):401-406.

53. Colhoun HM, Ginsberg HN, Robinson JG, et al. No effect of PCSK9 inhibitor alirocumab on the incidence of diabetes in a pooled analysis from 10 ODYSSEY phase 3 studies. Eur Heart J. 2016;37 (39):2981-2989.

54. Schmidt AF, Swerdlow DI, Holmes MV, et al. PCSK9 genetic variants and risk of type 2 diabetes: a mendelian randomisation study. Lancet Diabetes Endocrinol. 2016.

55. Lotta LA, Sharp SJ, Burgess S, et al. Association between lowdensity lipoprotein cholesterol-lowering genetic variants and risk of type 2 diabetes: a meta-analysis. JAMA. 2016;316(13):1383-1391.

56. Ference BA, Robinson JG, Brook RD, et al. Variation in PCSK9 and HMGCR and risk of cardiovascular disease and diabetes. N Engl J Med. 2016;375(22):2144-2153.

-. This manuscript reports a prediction to develop type II diabetes based on PCSK9 polymorphisms related to LDL-cholesterol reduction.

57. Kazi DS, Moran AE, Coxson PG, et al. Cost-effectiveness of PCSK9 inhibitor therapy in patients with heterozygous familial hypercholesterolemia or atherosclerotic cardiovascular disease. JAMA. 2016:316(7):743-753. 